\title{
A 67-year-old man with facial droop, ataxia and vertigo
}

\author{
Laila Nasser MD BHSc, Siobhan Deshauer MD BMus, Kyla Caners MD, Amitabha Chakraborty MD
}

Cite as: CMAJ 2020 June 8;192:E626-9. doi: 10.1503/cmaj.190474

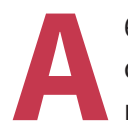

67-year-old white man presented to the emergency department with a 10-day history of left-sided facial numbness, vertigo and nausea, along with new-onset gait imbalance. He had been treated for 1 week with prednisone (45 mg/d) and amoxicillin (500 mg 3 times daily) for suspected Bell palsy and concomitant sinus infection.

The patient's medical history included hypercholesterolemia, colon cancer resection without recurrence (9 years previously) and smoking. His medications included rosuvastatin and acetylsalicylic acid for primary prevention. He had no history of travel or weight loss, fever or night sweats.

On examination, the patient had head deviation to the left in lateral flexion at rest (Figure 1). He had left-sided facial asymmetry sparing the forehead (Figure 2) and decreased facial sensation over the $V_{2}-V_{3}$ distribution. He also had ptosis and an inability to close his left eye, with associated horizontal nystagmus on rightward gaze (Figure 3). His pupils were equal and reactive to light, with normal visual acuity and visual field testing. Hearing was decreased on the left. His gait was ataxic with a wide base and he could not achieve tandem gait. Patellar reflex was brisk and plantar response upgoing on the right. The remaining neurologic examination was normal, including midline tongue, palate rise, cerebellar finger-to-nose testing, tone, sensation and motor testing. Findings on cardiovascular, respiratory, lymphatic and abdominal examinations were normal, with no evidence of dysautonomia.

On initial blood work, the patient's complete blood count, electrolytes and creatinine were within normal limits. His electrocardiogram showed normal sinus rhythm.

\section{Which syndrome is most in keeping with this patient's deficits?}
a. Weber syndrome
b. Partially treated Bell palsy
c. Benedikt syndrome
d. Lateral medullary syndrome

The patient presented with left-sided facial droop and numbness, ptosis, horizontal nystagmus and ataxia, which are

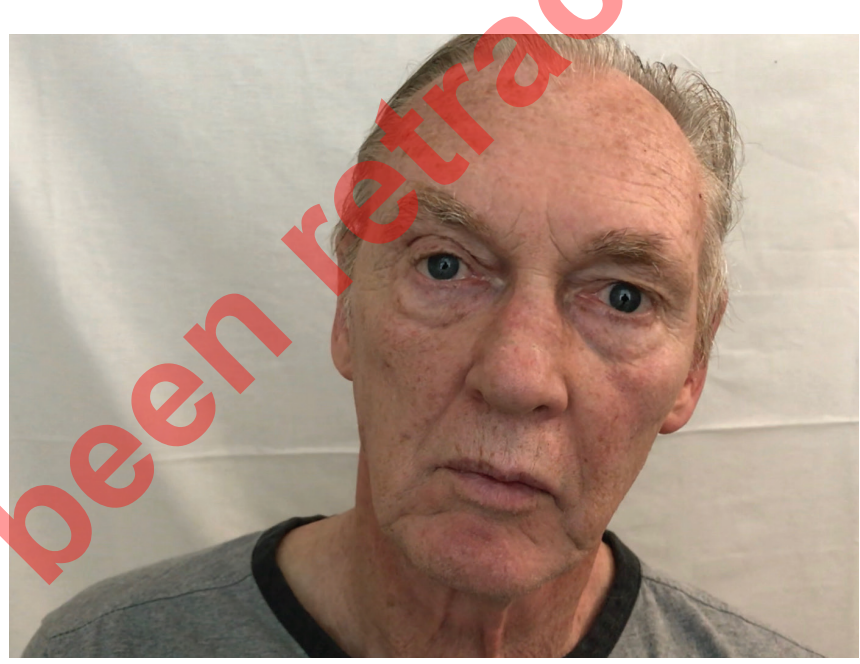

Figure 1: Head deviation to the left in lateral flexion at rest in a 67 -yearold man with a 10-day history of left-sided facial numbness, vertigo and nausea, and new onset of gait imbalance.

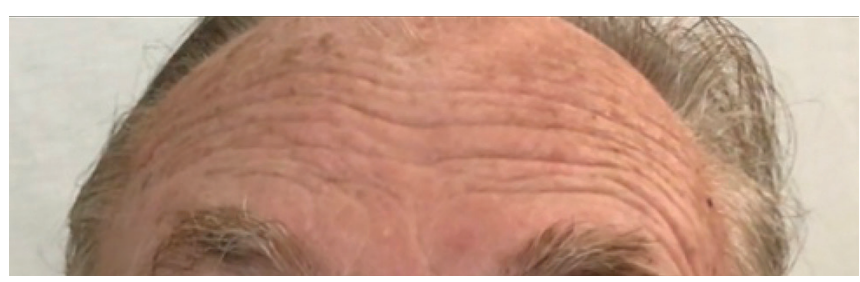

Figure 2: Symmetric forehead with raising of the eyebrows.

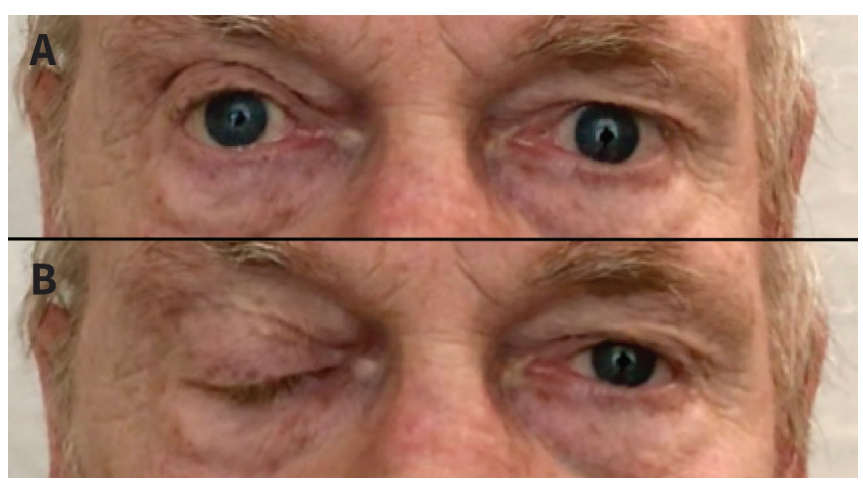

Figure 3: Ptosis of the left eye (A) and inability to close left eye with blinking (B). 
pathognomonic for lateral medullary syndrome (or Wallenberg syndrome) (d). Typical features include ipsilateral loss of pain, temperature and sensation in the face; ipsilateral Horner syndrome; and contralateral body hemianesthesia, vertigo, ataxia and nausea. ${ }^{1}$ The patient was correctly identified as having a central cause of seventh nerve palsy (rather than peripheral), as his motor deficit spared his forehead. The forehead receives bilateral cortical input as a result of multiple dorsal corticobulbar tract crossings in the brainstem.

The patient's upgoing plantar response and brisk reflexes indicate an upper motor neuron lesion, also suggesting a central cause. ${ }^{1}$ Sensory deficit in the ipsilateral face is related to ischemia of the trigeminal nucleus, where vertigo and ataxia are a result of an insult to the vestibular nuclei (Figure 4). The patient's resulting head tilt is compensation for dysfunction of ocular alignment.

Bell palsy (b) is a peripheral cause of seventh nerve palsy and is differentiated from a central cause by motor involvement of the forehead and lower face. Partial treatment would not change the distribution of the patient's facial weakness, and his constellation of other symptoms (i.e., gait disturbance, brisk reflexes) is not in keeping with peripheral palsy. ${ }^{1}$

Both (a) Weber and (c) Benedikt syndromes have contralateral hemiplegia (rather than sensory loss) and contralateral gait disturbance (rather than vertigo and ataxia). ${ }^{2}$ Ipsilateral Horner syndrome can occur in Weber and Benedikt syndromes from posterior circulation infarction disrupting sympathetic output, and also in Bell palsy. ${ }^{2}$

Presuming a central cause, a computed tomography (CT) angiogram was ordered in the emergency department to rule out a stroke. The CT scan had no abnormalities and the patient was admitted to the internal medicine service for further investigation, including magnetic resonance imaging (MRI) for further evaluation of the brainstem and posterior fossa. In addition to a demyelinating or neoplastic lesion observed in the pons (Figure 5), a small remote right cerebellar infarct was visible on MRI, with moderate cerebral atrophy in the deep white matter, suggestive of chronic microangiopathic ischemic changes or demyelination (not shown).

Further blood work showed a normal $B_{12}$ level, erythrocyte sedimentation rate $(10 \mathrm{~mm} / \mathrm{h})$, and negative HIV and Venereal Disease Research Laboratory test screening. No abnormalities were found on cardiac telemetry and echocardiogram.

\section{What is the most likely diagnosis?}

a. Posterior circulation ischemic stroke

b. Primary central nervous system lymphoma

c. First presentation of multiple sclerosis

d. Central nervous system vasculitis

Posterior circulation ischemic strokes (a) remain the most common cause of lateral medullary syndrome. Given the patient's age, sex and smoking history, a thromboembolic event must be ruled out first. ${ }^{3}$

This patient does not have typical risk factors for central nervous system lymphoma (e.g., immunodeficiency such as from HIV), but is in the right age group (45-65 yr) and he presented with neurologic changes in the context of a new brain lesion. This diagnosis (b) cannot yet be ruled out. ${ }^{4}$

Multiple sclerosis (c) is a multifocal demyelinating disease of the central nervous system, which typically presents in adults aged 20-50 years, more often in women than men. A small proportion of those affected present at age older than 60 years. ${ }^{5}$ Clinically isolated syndromes of multiple sclerosis typically affect

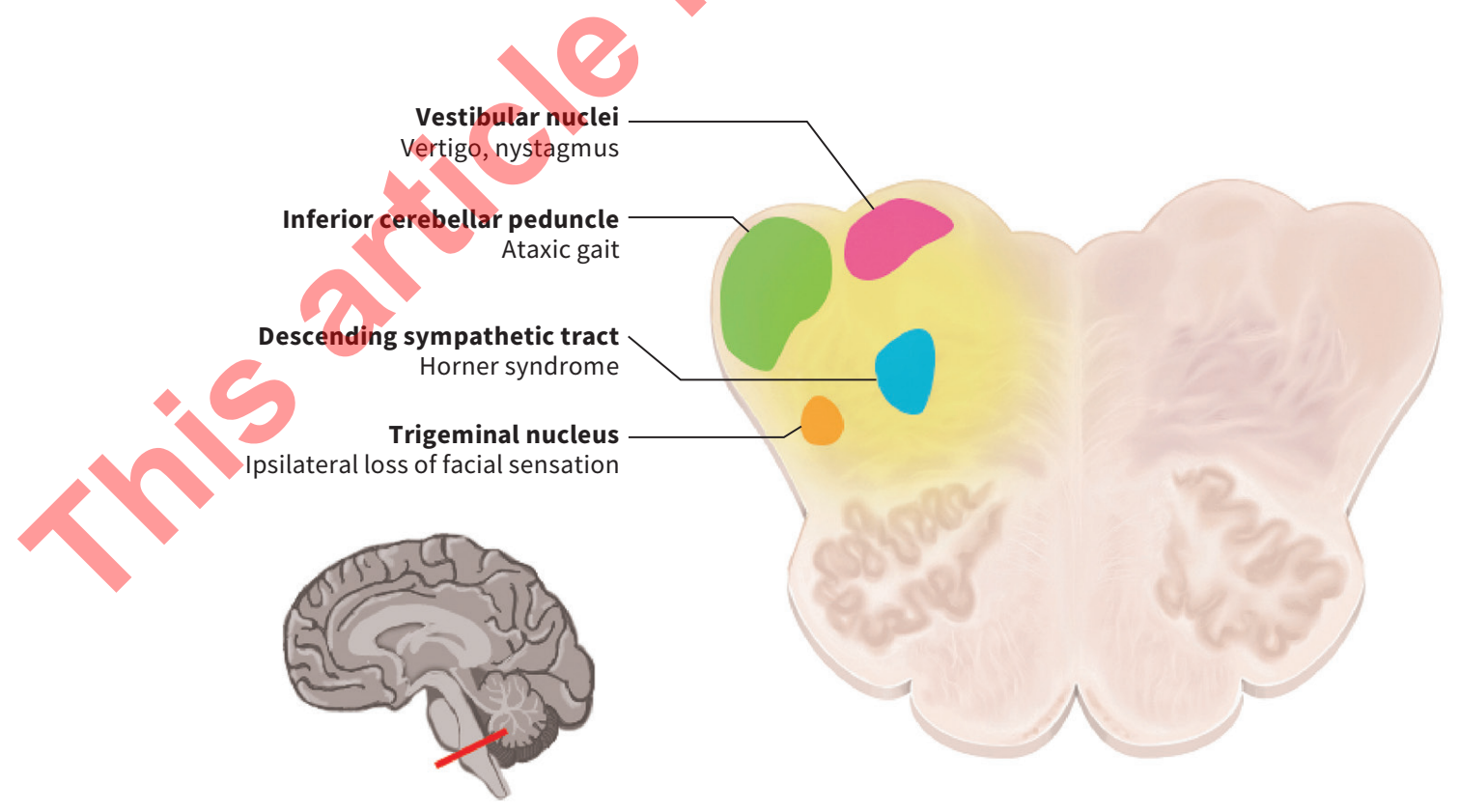

Figure 4: Pathoanatomy of lateral medullary syndrome. Credit: Amy Ajay. 
the optic nerve, cerebellum or spinal cord. ${ }^{2}$ With only 1 lesion on MRI and no history of other neurologic deficits, the patient did not yet meet the McDonald criteria for diagnosis of multiple sclerosis, which stipulates that demyelinating lesions must be disseminated in space (in the central nervous system) or in time
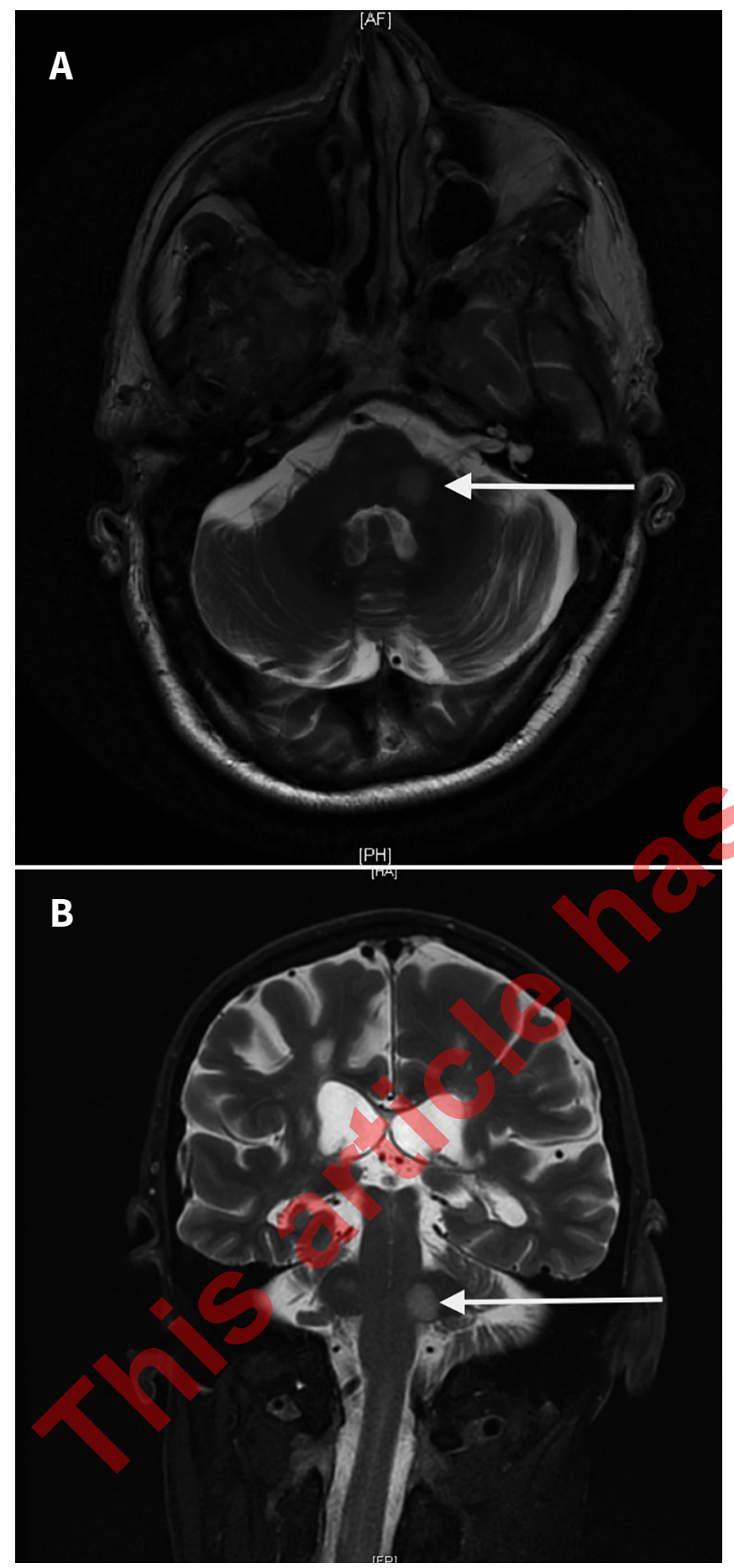

Figure 5: Magnetic resonance imaging scan of the brain in (A) axial and (B) coronal views. Both display a 1-cm well-circumscribed hyperintense $T_{2}$ signal in the left pons (arrows), suggestive of a demyelinating or neoplastic lesion, with no perilesional edema or mass effect. Sequences acquired were $T_{1}, T_{2}$, fluid-attenuated inversion recovery (FLAIR) and diffusion-weighted imaging (DWI). (multiple attacks). ${ }^{5,6}$ However, the presentation of multiple sclerosis can be acute and mimic stroke, and cannot yet be ruled out.

Primary central nervous system vasculitis (d) is an uncommon condition that occurs more often in men; mean age at diagnosis is 50 years. ${ }^{1}$ It classically presents with a long prodrome, nonspecific neurologic symptoms and normal erythrocyte sedimentation rate. Given the abrupt onset of this patient's symptoms and the rarity of the condition, it is not the most likely diagnosis.

A cardioembolic event was unlikely, given the normal telemetry and echocardiogram. With an MRI suggesting a demyelinating process, and having ruled out a posterior stroke (a), the most correct answer of the options presented above is (c), first presentation of multiple sclerosis.

\section{What is the next best diagnostic step?}

a. Lumbar puncture

b. Magnetic resonance imaging of the spine

c. Visual evoked potentials

d. Magnetic resonance imaging of the brain with gadolinium

Given the results of our patient's brain MRI, the differential diagnosis included a neoplastic lesion or demyelinating disease. The next best test is an MRI brain scan with gadolinium (d), as multiple sclerosis lesions in the brain are transiently enhanced in the weeks surrounding illness (more so than in the spine). ${ }^{7}$ However, this test was not readily available in our acute setting, so we undertook a lumbar puncture (a) to obtain cerebrospinal fluid and ordered an MRI of the spine (b) to complete this patient's workup. The cerebrospinal fluid was used to test for the presence of oligoclonal banding consistent with multiple sclerosis, the presence of neoplastic cells and for flow cytometry to rule out central nervous system lymphoma. We checked for further demyelinating lesions suggestive of multiple sclerosis with an MRI of the spine. Visual evoked potentials are classically used to detect demyelination and optic neuritis but are not used in isolation to make the diagnosis of multiple sclerosis. ${ }^{8}$

The results of the lumbar puncture were unremarkable except for a slightly elevated protein level of 0.68 (normal $<0.45$ ) $\mathrm{g} / \mathrm{L}$ in the cerebrospinal fluid. Oligoclonal banding was negative. Flow cytometry did not show evidence of clonal T- or B-cell populations.

The inpatient neurology and neuro-ophthalmology services were consulted and ruled out neurosarcoidosis with a normal ocular examination, negative angiotensin-converting enzyme level, normal calcium level and unremarkable chest radiography. Fabry disease was excluded with normal genetic testing.

A month after the initial MRI of the patient's head, a repeat MRI enhanced with gadolinium was performed. It displayed incomplete ring enhancement in the previously visualized left pontine lesion as well as in the right periventricular white matter, suggestive of active demyelination. These findings indicated that the patient now met the McDonald criteria for multiple sclerosis, with dissemination in space and time.

At the time of the repeat MRI of the head, the patient's symptoms were improving but he still had ongoing disturbances to his balance, and his facial droop and eye findings were still present. 


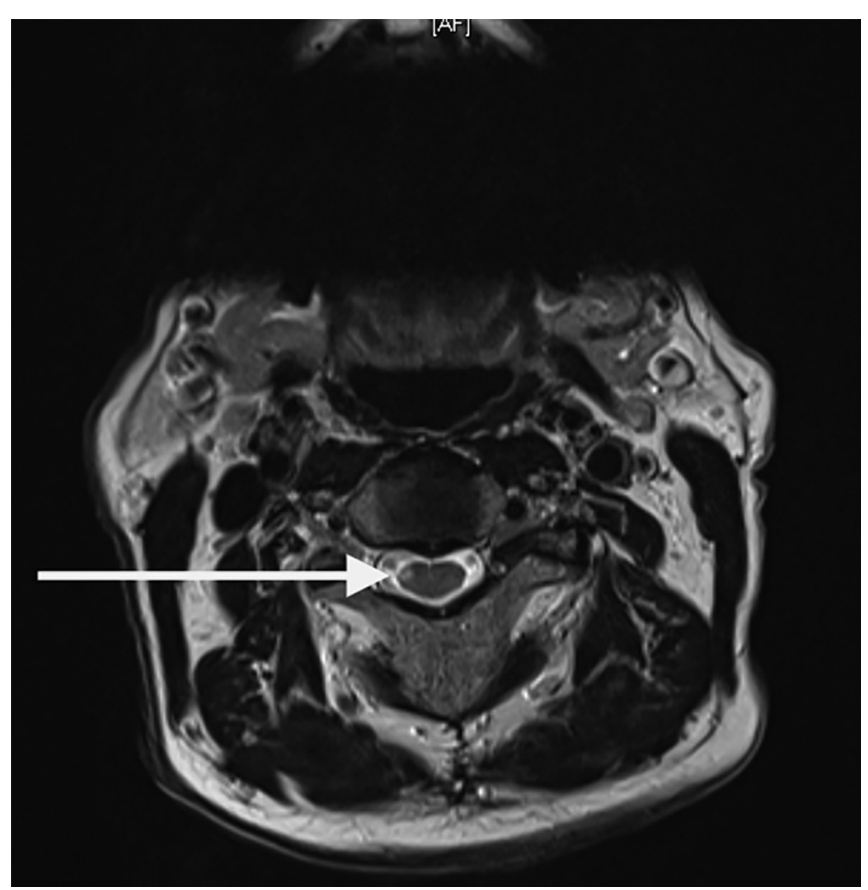

Figure 6: Magnetic resonance imaging scan of the cervical spine 3 months after presentation, showing a $T_{2}$ lesion at the $\mathrm{C} 1-\mathrm{C} 2$ level (arrow).

Three months later, an MRI of the spine showed a small, nonenhancing demyelinating lesion at the C1-C2 level (Figure 6).

At a follow-up appointment 5 months after the patient's initial presentation, the diagnosis of an atypical presentation of multiple sclerosis was confirmed by neurology. The patient continues to be monitored regularly by the multiple sclerosis clinic.

\section{Discussion}

This case highlights an atypical onset of multiple sclerosis. The patient presented with a new onset of lateral medullary syndrome; however, it was only with further testing that multiple sclerosis was diagnosed. The most common association with lateral medullary syndrome is posterior inferior cerebellar artery stroke, but there are a number of other causes. ${ }^{5}$ The first step is to recognize the constellation of symptoms including facial nerve palsy and central vertigo, then pursue a broad differential of intracranial pathology that includes ischemia, central nervous system lymphoma, vasculitis and multiple sclerosis (as an atypical cause).

The final diagnosis in this case was multiple sclerosis, fulfilling McDonald criteria with the patient having 1 "attack" and objective clinical evidence of an indicative lesion on MRI in 2 areas of the central nervous system (periventricular and left pons). ${ }^{6} \mathrm{~A}$ multicentre retrospective study examined MRI criteria for patients with clinically isolated syndromes and thought to have multiple sclerosis. ${ }^{7}$ It found that the presence of lesions that are disseminated in both time and space from 2 MRI scans has a higher specificity and risk for clinically definite multiple sclerosis than lesions meeting either dissemination in time or dissemination in space criteria alone. The study found that multiple sclerosis criteria can be met in patients presenting with clinically isolated syndromes, as in our patient.
The lesson from this case is that demyelinating disease can present at an atypical age, and that central vertigo or a facial palsy can be the first presentation. ${ }^{9,10}$ It is crucial to recognize the features of a possible lateral medullary syndrome and order the appropriate imaging to investigate for stroke and other central causes, such as multiple sclerosis, when warranted.

\section{References}

1. Walls R, Hockberger R, Gausche-Hill M. Rosen's emergency medicine: concepts and clinical practice. 9th ed. Toronto: Elsevier Canada; 2017.

2. Snell RS. Clinical neuroanatomy. 7th ed. Philadelphia: Lippincott Williams \& Wilkins; 2010.

3. Caplan L. Posterior circulation cerebrovascular syndromes. UpToDate; 2017 [updated 2018 Apr. 30].

4. Ho KL. Uncommon causes of the lateral medullary syndrome. Arch Neurol 1980;37:669-70.

5. Sacco RL, Freddo L, Bello JA, et al. Wallenberg's lateral medullary syndrome: clinical-magnetic resonance imaging correlations. Arch Neurol 1993;50:609-14.

6. Thompson AJ, Banwell BL, Barkhof F, et al. Diagnosis of multiple sclerosis: 2017 revisions of the McDonald criteria. Lancet Neurol 2018;17:162-173.

7. Filippi M, Preziosa $P$, Banwell BL, et al. Assessment of lesions on magnetic resonance imaging in multiple sclerosis: practical guidelines. Brain 2019; 142:1858-75.

8. Walsh P, Kane N, Butler S. The clinical role of evoked potentials. J Neurol Neurosurg Psychiatry 2005;76(suppl 2):ii16-22.

9. Swanton JK, Rovira A, Tintore M, et al. MRI criteria for multiple sclerosis in patients presenting with clinically isolated syndromes: a multicentre retrospective study. Lancet Neurol 2007;6:677-86.

10. Qiu W, Wu J-S, Carroll WM, et al. Wallenberg syndrome caused by multiple sclerosis mimicking stroke. J Clin Neurosci 2009;16:1700-2.

\section{Competing interests: None declared.}

This article has been peer reviewed.

The authors have obtained patient consent.

Affiliations: Divisions of Emergency Medicine (Nasser, Caners) and Internal Medicine (Deshauer, Chakraborty), McMaster University; Hamilton Health Sciences (Nasser, Deshauer, Caners, Chakraborty).

Contributors: Kyla Caners and Amitabha Chakraborty contributed to the conception and design of the work. Laila Nasser and Siobhan Deshauer drafted the manuscript. All of the authors revised the manuscript critically for important intellectual content, gave final approval of the version to be published and agreed to be accountable for all aspects of the work.

Acknowledgements: The authors thank Amy Ajay BHSc (McMaster University) for the creation of Figure 4 (Pathoanatomy of Lateral Medullary Syndrome).

Disclaimer: Siobhan Deshauer is the daughter of Dorian Deshauer, who is a deputy editor with CMAJ. Dorian Deshauer was not involved in the editorial decision-making for this article.

Correspondence to: Laila Nasser, laila.nasser@medportal.ca

CMAJ invites submissions to "What is your call?" Clinical details (including images) are presented with a multiple-choice question about the diagnosis. The answer and a brief discussion of the condition follow. We specifically invite submissions illustrating common or important radiographic and electrocardiographic diagnoses of appeal to a general audience. We require authors to obtain consent from the patient for publication of his or her story. Submit manuscripts online at http://mc.manuscriptcentral.com/cmaj 